\title{
Electron Correlation Microscopy Measurements of Metallic Glass Surface Dynamics
}

\author{
Debaditya Chatterjee and Paul Voyles
}

University of Wisconsin - Madison, Madison, Wisconsin, United States

Metallic glass forming liquids exhibit spatial and temporal heterogeneity in their structure and relaxation behavior. In the supercooled state, it has been shown that these spatial heterogeneities are at the nanometer-scale [1]. Electron correlation microscopy (ECM) uses electron nanodiffraction at elevated temperatures in a transmission electron microscope (TEM) to study the dynamics of fluctuating systems like supercooled metallic glass forming liquids [2]. We have performed ECM in the tilted dark-field (TDF) TEM imaging mode which can measure relaxation times from many positions in parallel and allows us to derive spatial maps of relaxation time with sub-nanometer spatial resolution [3].

We have applied ECM to study near-surface layer dynamics in metallic glass forming liquids as a function of temperature using in situheating. Previous ECM studies have revealed that the near-surface layer relaxation dynamics in $\mathrm{Pt}_{57.5} \mathrm{Cu}_{14.7} \mathrm{Ni}_{5.3} \mathrm{P}_{22.5}$ metallic glass nanowires are an order of magnitude faster than bulk dynamics at the same temperature. We have investigated dynamics of the near-surface layer by performing ECM experiments with higher spatial resolution and at temperatures lower than the bulk glass transition temperature, where the surface layer dynamics are still active but the bulk is vitrified. We have also studied the effect of the presence of a confining layer on the nanowire surface on the near-surface layer dynamics.

Fig 1(a) shows a relaxation time map of a nanowire heated in situ at $227^{\circ} \mathrm{C}$. We can see a $\sim 1 \mathrm{~nm}$ layer with faster dynamics near the surface of the nanowire. This fast layer is hypothesized to play an important role in determining the mechanism of crystal nucleation from the supercooled liquid [3]. Fig 1(b) shows the variation of bulk and near-surface relaxation times as a function of temperature. Extrapolating the surface relaxation time trendline to the bulk relaxation time at the literature glass transition temperature reveals a surface glass transition temperature of $487 \mathrm{~K}, 20 \mathrm{~K}$ lower than the bulk.

Fig 2 shows the effect of a surface confinement layer on relaxation dynamics in nanowires coated with a $\sim 10 \mathrm{~nm}$ layer of amorphous carbon. Fig 2(a) shows the DF-TEM image of a nanowire coated with amorphous carbon. Figs 2(b) and (c) show relaxation time maps in amorphous carbon coated nanowires heated in situ at $242^{\circ} \mathrm{C}$ and $246^{\circ} \mathrm{C}$ respectively. The fast near-surface layer is not observed. This result indicates that access to the free volume available at the surface is responsible for the enhanced mobility, since removing access suppresses the fast surface layer.

Experiments are being developed to correlate the local structure with relaxation dynamics in these systems with sub-nanometer scale spatial resolution. These would involve acquisition of fully resolved 5dimensional datasets, intensity as a function of position $(x, y)$, scattering vector $\left(k_{x}, k_{y}\right)$ and time $t$. A next generation fast camera capable of running at $100 \mathrm{kHz}$ or faster is in development to enable these experiments [4]. 

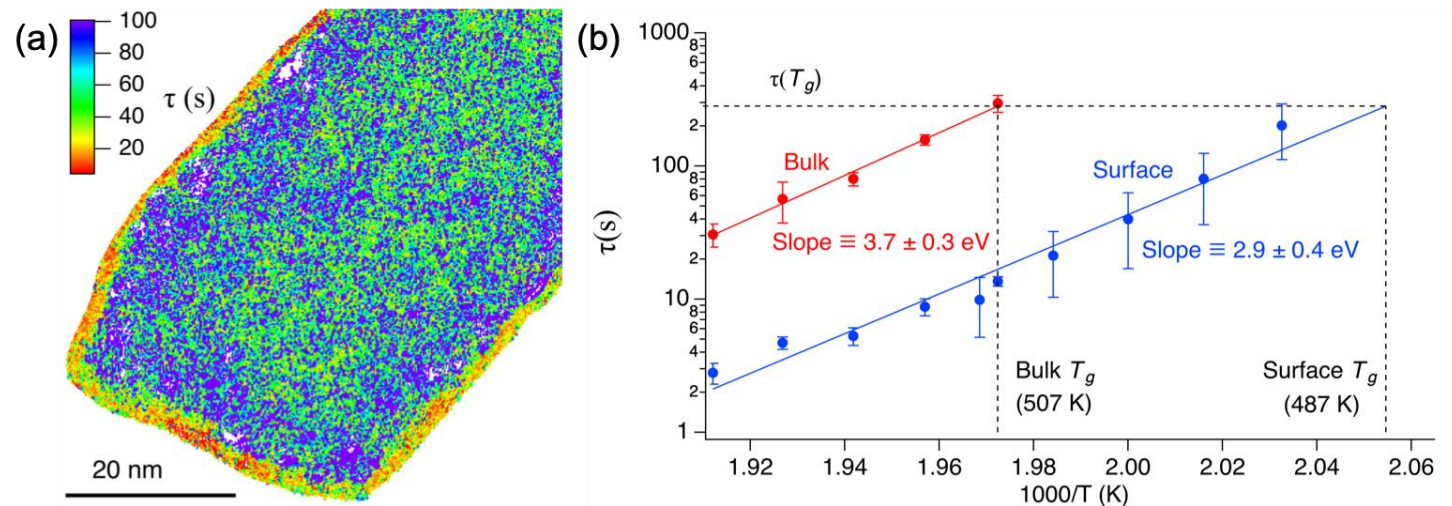

Figure 1. (a) Relaxation time $(\tau)$ map of a nanowire heated in situ at $227^{\circ} \mathrm{C}$ showing a near-surface layer with faster relaxation dynamics compared to the bulk, (b) The mean structural relaxation time for the nanowire interior (bulk) and the near-surface layer. The error bars are the standard deviation of the measured relaxation time measured at different pixels. Fitting to the Arrhenius form yields activation energies of $3.7 \pm 0.3 \mathrm{eV}$ for the bulk and $2.9 \pm 0.4 \mathrm{eV}$ for the near-surface layer. The near-surface layer glass transition temperature is suppressed by $20 \mathrm{~K}$ compared to the bulk.
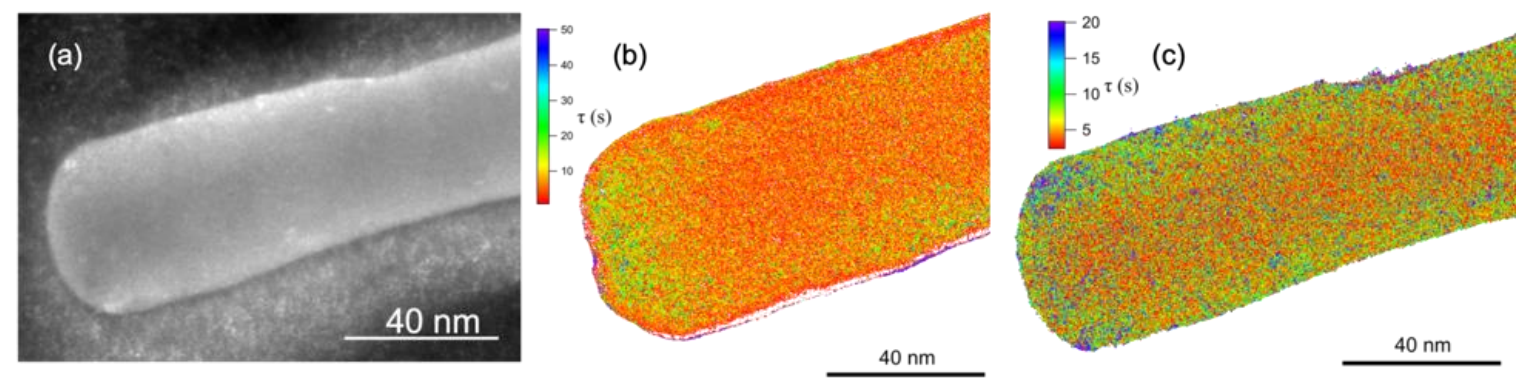

Figure 2. (a) DF-TEM image showing a nanowire coated with a $10 \mathrm{~nm}$ layer of sputtered amorphous carbon, (b-c) Relaxation time maps of amorphous carbon coated nanowires heated in situ at $242^{\circ} \mathrm{C}$ and $246^{\circ} \mathrm{C}$ respectively. The fast near-surface layer is not observed.

\section{References}

[1] Ediger, M. D. Annu. Rev. Phys. Chem. 51, 99-128 (2000)

[2] P Zhang et al., Ultramicroscopy 178 (2017), p. 125

[3] P Zhang et al., Nature Communications 9 (2018), p. 1129.

[4] This research is support by the US National Science Foundation (DMR-1807241). 